\title{
Ultrasound Evaluation of Fluid in Knee Recesses at Varying Degrees of Flexion
}

\author{
P. MANDL, ${ }^{1}$ M. BROSSARD, ${ }^{2}$ P. AEGERTER,${ }^{2}$ M. BACKHAUS,${ }^{3}$ G. A. BRUYN, ${ }^{4}$ \\ I. CHARY-VALCKENAERE, ${ }^{5}$ A. IAGNOCCO ${ }^{6}{ }^{\text {E. FILIPPUCCI, }}{ }^{7}$ J. FREESTON, ${ }^{8}$ F. GANDJBAKHCH, ${ }^{9}$ \\ S. JOUSSE-JOULIN, ${ }^{10}$ I. MÖLLER, ${ }^{11}$ E. NAREDO,$^{12}$ W. A. SCHMIDT, ${ }^{13}$ M. SZKUDLAREK, ${ }^{14}$ L. TERSLEV, ${ }^{15}$ \\ R. J. WAKEFIELD, ${ }^{8}$ A. ZAYAT, ${ }^{8}$ M. A. D'AGOSTINO, ${ }^{2}$ AND P. V. BALINT ${ }^{16}$
}

Objective. Various methods are utilized in daily practice to obtain optimal information on effusion in the knee. Our aim is to investigate which scanning position provides the best information about synovial fluid in the knee by using ultrasound and to evaluate the magnitude of difference for measuring synovial fluid in 3 major recesses (suprapatellar, medial parapatellar, and lateral parapatellar) of the knee according to various degrees of flexion.

Methods. Sonographers in 14 European centers documented bilateral knee joint ultrasound examinations on a total of 148 knee joints. The largest sagittal diameter of fluid was measured in scans corresponding to the 3 major recesses at different $\left(0^{\circ}, 15^{\circ}, 30^{\circ}, 45^{\circ}, 60^{\circ}\right.$, and $\left.90^{\circ}\right)$ degrees of flexion of the knee. The difference of measurement of effusion according to transducer position, knee position, and the interaction between them was investigated by analysis of variance followed by Tukey's test.

Results. No correlation was noted between patient characteristics and ultrasound detection of effusion. The sagittal diameter of synovial fluid in all 3 recesses was greatest at $30^{\circ}$ flexion. Analysis of variance and Tukey's test revealed that the suprapatellar scan and $30^{\circ}$ flexion is the best combination for detecting effusion as confirmed by receiver operator characteristic curve analysis.

Conclusion. The suprapatellar scan of the knee in $30^{\circ}$ flexion was the most sensitive position to detect fluid in knee joints. Sagittal diameter of fluid in all 3 recesses increased with the knee in the $30^{\circ}$ flexed position as compared to the extended position.

\section{INTRODUCTION}

The knee joint is commonly affected in inflammatory joint conditions. Several studies have suggested that ultrasonography is superior to clinical examination in determining the presence and localization of knee joint effusion (1-5). Despite these findings, there is a lack of guidelines-

${ }^{1}$ P. Mandl, MD, PhD: Medical University of Vienna, Vienna, Austria and National Institute of Rheumatology and Physiotherapy, Budapest, Hungary; ${ }^{2}$ M. Brossard, MSc, P. Aegerter, MD, PhD, M. A. D'Agostino, MD, PhD: Université de Versailles-St.-Quentin-en-Yvelines, AP-HP, Ambroise Paré Hospital, Boulogne-Billancourt, France; ${ }^{3} \mathrm{M}$. Backhaus, MD, PhD: Charité University Hospital, Berlin, Germany; ${ }^{4}$ G. A. Bruyn, MD, PhD: Medisch Centrum IJsselmeerziekenhuizen, Lelystad, The Netherlands; ${ }^{5}$ I. Chary-Valckenaere, MD, PhD: University of Nancy, Le Brabois Hospital, Nancy, France; ${ }^{6} \mathbf{A}$. Iagnocco, MD: Sapienza University, Rome, Italy; ${ }^{7} \mathrm{E}$. Filippucci, MD, PhD: Universitá Politecnica delle Marche, Ancona, Italy; ${ }^{8}$ J. Freeston, MA, MRCP, R. J. Wakefield, BM, MD, MRCP, A. Zayat, MD: University of Leeds and NIHR Leeds Musculoskeletal Biomedical Research Unit, Leeds, UK; ${ }^{9} \mathrm{~F}$. Gandjbakhch, MD: CHU Pitie Salpetriere Hospital, Paris, France; ${ }^{10}$ S. Jousse-Joulin, MD: CHU Cavale Blanche, Brest, France; ${ }^{11}$ I. Möller, MD: Instituto Poal de Reumatologia- regarding the proper positioning of the joint during both clinical and ultrasound examination. The European League Against Rheumatism (EULAR) guidelines for performing musculoskeletal ultrasound examination recommend examining the knee joint in either the flexed or extended position (6). Voluntary quadriceps contraction has been utilized as a means to increase sensitivity of

Hospital Platon, Barcelona, Spain; ${ }^{12}$ E. Naredo, MD: Hospital Universitario Severo Ochoa, Madrid, Spain; ${ }^{13}$ W. A. Schmidt, MD, PhD: Medical Centre for Rheumatology Berlin-Buch, Berlin, Germany; ${ }^{14}$ M. Szkudlarek, MD, PhD: University of Copenhagen Hospital at Køge, Køge, Denmark; ${ }^{15}$ L. Terslev, MD, PhD: Copenhagen University Hospital at Glostrup, Copenhagen, Denmark; ${ }^{16}$ P. V. Balint, MD, PhD, FRCP: National Institute of Rheumatology and Physiotherapy, Budapest, Hungary.

Dr. Backhaus has received consultant fees, speaking fees, and/or honoraria (more than $\$ \mathbf{1 0 , 0 0 0}$ each) from Abbott, Merck Sharp \& Dohme, Pfizer, Roche, and Bristol-Myers Squibb.

Address correspondence to $\mathrm{P}$. Mandl, MD, PhD, Division of Rheumatology, University of Vienna, 18-20 Währinger Gürtel, 1090 Vienna, Austria. E-mail: mandlpeter@yahoo.com.

Submitted for publication June 28, 2011; accepted in revised form January 3, 2012. 


\section{Significance \& Innovations}

- This international multicenter study has shown that the position of the knee influences the sensitivity of ultrasound for detecting synovial fluid in knee joints.

- The suprapatellar scan of the knee in $30^{\circ}$ flexion was found to be the most sensitive position to detect synovial fluid in knee joints.

- Full ultrasound evaluation of the knee should include the dynamic examination (knee flexed from $0^{\circ}$ to $90^{\circ}$ ) of the 3 major recesses of the knee, which provides the best information on synovial fluid in the knee joint.

detecting synovial fluid in the suprapatellar recess in osteoarthritic knee joints where effusion could not be visualized in the standard, extended position without quadriceps contraction (7).

The extent of varying degrees of flexion and its effect on the detection of synovial fluid in multiple recesses of the knee has previously not been investigated. The suprapatellar recess or bursa is a pouch formed by the articular capsule of the knee possessing its own synovial lining (8), while the medial parapatellar and lateral parapatellar recesses are extensions thereof in the medial parapatellar and lateral parapatellar areas, respectively. The parapatellar recesses are recommended among the standard scans of the knee in the guidelines on the use of sonography in rheumatology (6) and have been utilized for individual measurement of fluid volume in the knee by several investigations utilizing ultrasound, as well as other imaging modalities (9-12). We therefore evaluated the magnitude of difference for measuring synovial fluid in these major recesses (suprapatellar, medial parapatellar, and lateral parapatellar recess) of the knee according to various degrees of flexion. We then investigated which of these positions best influences the detection of abnormal fluid after performing a complete ultrasonographic examination of the knee.

\section{PATIENTS AND METHODS}

In this international multicenter study, 18 rheumatologists with several years of experience in musculoskeletal ultrasound were asked to conduct ultrasound examinations over a period of 1 month in their respective practices on patients with knee joint symptoms (involving at least 1 knee). Exclusion criteria included patients who were unable to perform knee flexion or could not extend their knees completely, patients who underwent knee surgery in the examined knee, and patients without symptoms in either knee. Sonographers documented the sex, age, and diagnosis of the patients. Anonymous data were entered into case report sheets and analyzed in a single center. Ethics approval was not obtained for this study since all included patients were referred to the ultrasound examination for reasons other than participation in the present study and because the standard ultrasound examination of the knee already includes the scans and knee positions performed within the framework of this project.

Ultrasound. Gray-scale and power Doppler ultrasound examination of each knee was performed at the following 3 major suprapatellar pouch recesses: midline suprapatellar, medial parapatellar, and lateral parapatellar (Figures 1A, B, and C). Examination of the midline suprapatellar recess was carried out in the sagittal plane at midline, while that of the medial parapatellar and lateral parapatellar recess was carried out in the midpatellar transverse plane $90^{\circ}$ medial and lateral from midline, respectively. Scans of the 3 recesses were performed at $0^{\circ}, 15^{\circ}, 30^{\circ}, 45^{\circ}$, $60^{\circ}$, and $90^{\circ}$ of flexion of the knee joint, yielding a total number of 18 scans for each knee joint.

Degree of flexion was established with the use of a standard goniometer. The knee was unsupported by external support (e.g., foam block) during the procedure. For each examination generous amounts of gel were applied to the knee and each sonographer took care in applying only

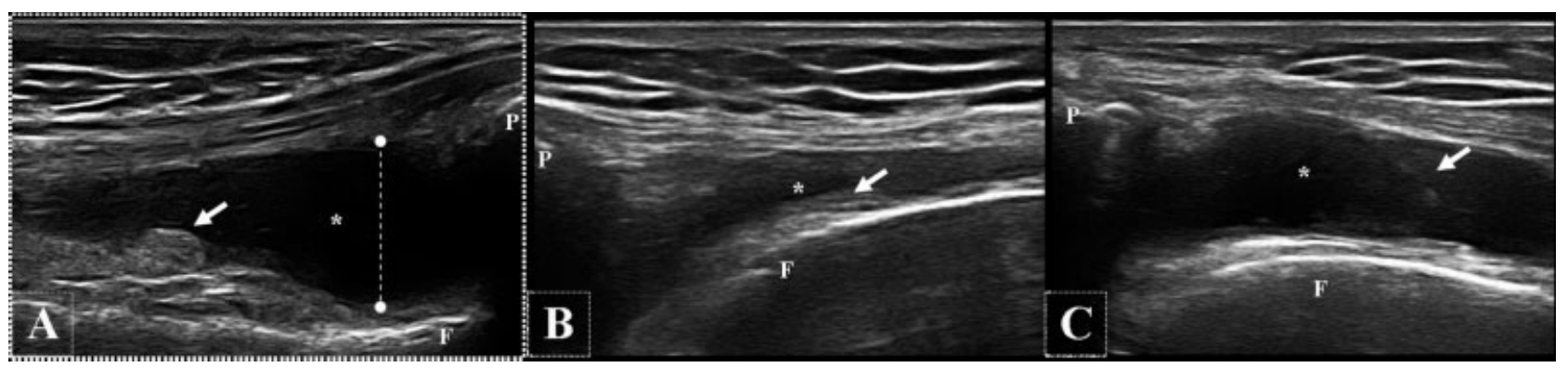

Figure 1. Sonographic examination of major recesses of the knee: A, suprapatellar recess, sagittal plane; B, medial parapatellar recess, midpatellar transverse plane; and $\mathbf{C}$, lateral parapatellar recess, midpatellar transverse plane. ${ }^{*}=$ effusion; $\mathbf{F}=$ femur; $\mathbf{P}=$ patella; arrow $=$ synovial hypertrophy; dotted line = measurement of greatest diameter of fluid. 
minimal pressure to the transducer during the examination in order not to displace the fluid collection. Fluid collection was defined as an anechoic or hypoechoic area that is displaceable and does not exhibit Doppler signal according to the Outcome Measures in Rheumatology definition of synovial fluid (13). Sonographers used the following equipment: GE Logiq9, Esaote MyLab 70, MyLab 70 XVG, Esaote My Lab 25 Gold, Esaote Technos MPX, and Philips HDI 5000 with linear transducers with a frequency adapted to the examination of the knee (5$12 \mathrm{MHz}$ ).

Ultrasonographers performed a complete ultrasonographic examination of each knee in accordance with the EULAR guidelines (6). Moreover, for each recess in every examined angle of flexion, the absence or presence of synovial fluid and the greatest diameter of fluid perpendicular to the dorsal border of the fluid collection was recorded by using a sagittal scan. Based on the complete ultrasound examination, sonographers evaluated each joint either as normal or abnormal.

Statistical analysis. The several ultrasound scans of the suprapatellar, medial parapatellar, and lateral parapatellar recesses in the 6 knee positions $\left(0^{\circ}, 15^{\circ}, 30^{\circ}, 45^{\circ}\right.$, $60^{\circ}$, and $90^{\circ}$ ) yielded 18 measures of effusion diameter by knee for each subject. By taking the extended position $\left(0^{\circ}\right)$ of the knee as reference, we reduced the number of measures to 15 and we studied the difference of measurement according to knee position $\left(15^{\circ}-0^{\circ}, 30^{\circ}-0^{\circ}, 45^{\circ}-0^{\circ}\right.$, $60^{\circ}-0^{\circ}$, and $90^{\circ}-0^{\circ}$ ) and transducer position. In this way the variability due to the sonographer effect was also reduced.

To evaluate the proportion of variance explained by knee flexion, transducer position, and magnitude of change of the measurement of effusion according to the degree of flexion of the knee, we performed an analysis of the variance (ANOVA) explaining difference of measurement of effusion according to transducer position, knee position, and the interaction between them. This ANOVA was completed by Tukey's test in order to determine which combination of transducer position (recess) and knee position gave the highest difference of effusion, i.e., was the most sensitive for detecting the presence of effusion. The final judgment of knee abnormality at the end of the ultrasound examination was used as a dependent variable against which elementary measures (i.e., effusion at each position) were assessed.

Eighteen logistic regression models were performed to establish a relationship between the presence of effusion according to synovial fluid measured in each recess and knee position. Variables used in the models were selected among statistically significant variables obtained by univariate analysis. The 18 areas under the receiver operator characteristic curve (AUC-ROC) were used for evaluating the best discriminative combination for detecting fluid. Differences among each AUC were compared using 2,000 bootstrap samples, taking into account the repetition of

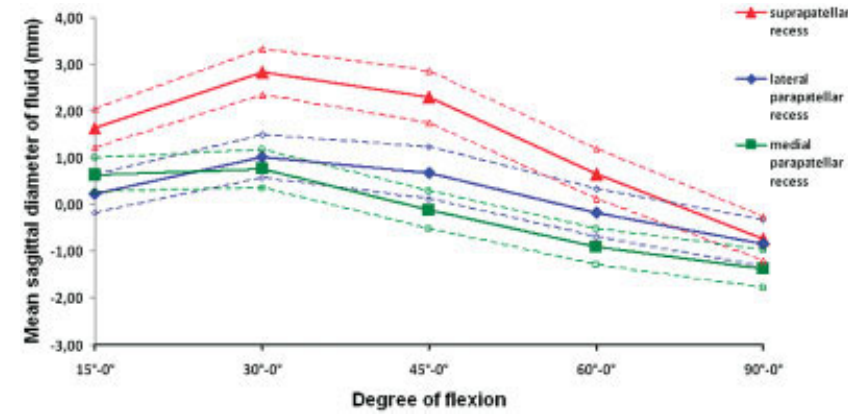

Figure 2. Sonographic evaluation of synovial fluid sagittal diameter of knee recesses at varying degrees of flexion. The confidence interaction $(95 \%)$ plot shows the mean sagittal diameter of synovial fluid for the 3 examined recesses for each combination between transducer and knee position, using the extended position $\left(0^{\circ}\right)$ as reference. Dotted lines represent confidence intervals for each of the corresponding recesses. For each recess, corresponding graphs are marked with matching colors. Color figure can be viewed in the online issue, which is available at http://online library.wiley.com/journal/10.1002/(ISSN)1529-0131.

measures on the same knees. For each of the 18 logistic regression models, the 3 best cutoff points corresponding to the maximal Youden index were chosen.

\section{RESULTS}

Demographics. A total of 148 knees of 77 patients were examined by 18 sonographers; 13 patients and an additional 6 knee joints were not evaluated due to missing or incomplete data. Fifty-three (69\%) patients were women. The mean \pm SD age was $58 \pm 15.7$ years (range 21-86 years). The subjects could be classified according to their diagnosis as follows: rheumatoid arthritis $=23$ patients; spondylarthritis (psoriatic arthritis, ankylosing spondylitis, undifferentiated oligoarthritis, and reactive arthritis) = 13 patients; crystal-associated synovitis $=3$ patients; osteoarthritis $=29$ patients; vasculitides and systemic lupus erythematosus $=7$ patients; and other (i.e., osteoporosis, septic arthritis) $=2$ patients.

Ultrasound data. Effusion was detected in 120 of 148 knees $(81 \%)$ during the ultrasound examination. Based on the full ultrasonographic examination of the knee, 106 of 148 knees (73\%) were classified as being abnormal. Sonographers judged final abnormality following a full knee ultrasound examination. Their evaluation was based on the presence/absence of a list of other abnormalities commonly detected in the knee joint by ultrasound, including synovial hypertrophy, Doppler activity, osteophytes, cartilage damage, etc. No correlation was noted between patient characteristics and ultrasound detection of effusion (data not shown).

Detection of effusion. ANOVA results showed that the magnitude of changes of sagittal diameter of synovial fluid measure varied significantly according to the angles of flexion $\left(P \leq 2 \times 10^{-16}\right)$, using the extended position $\left(0^{\circ}\right)$ as reference, according to transducer position $(P \leq 2 \times$ 


\begin{tabular}{|c|c|c|c|c|c|c|c|}
\hline Degree of flexion & AUC-ROC (95\% CI) & $\begin{array}{c}\text { Cutoff value, } \\
\text { mm }\end{array}$ & $\begin{array}{c}\text { Sensitivity } \\
\%\end{array}$ & $\begin{array}{c}\text { Specificity } \\
\%\end{array}$ & $\begin{array}{c}\text { Youden } \\
\text { index }\end{array}$ & LR+ & LR - \\
\hline \multicolumn{8}{|l|}{$0^{\circ}$} \\
\hline \multirow[t]{3}{*}{ Suprapatellar } & \multirow{3}{*}{$0.823(0.731-0.901)$} & 2.7 & 68.9 & 92.9 & 0.62 & 9.7 & 0.3 \\
\hline & & 2.4 & 73.1 & 85.7 & 0.59 & 5.1 & 0.3 \\
\hline & & 3 & 63.0 & 92.9 & 0.56 & 8.9 & 0.4 \\
\hline \multirow[t]{3}{*}{ Medial parapatellar } & \multirow[t]{3}{*}{$0.772(0.672-0.857)$} & 2.4 & 63.0 & 85.7 & 0.49 & 4.4 & 0.4 \\
\hline & & 2.7 & 59.7 & 85.7 & 0.45 & 4.2 & 0.5 \\
\hline & & 3 & 54.6 & 85.7 & 0.40 & 3.8 & 0.5 \\
\hline \multirow[t]{3}{*}{ Lateral parapatellar } & \multirow[t]{3}{*}{$0.839(0.757-0.910)$} & 2.4 & 77.3 & 85.7 & 0.63 & 5.4 & 0.3 \\
\hline & & 2.7 & 74.8 & 85.7 & 0.61 & 5.2 & 0.3 \\
\hline & & 1.8 & 81.5 & 78.6 & 0.60 & 3.8 & 0.2 \\
\hline \multicolumn{8}{|l|}{$15^{\circ}$} \\
\hline \multirow[t]{3}{*}{ Suprapatellar } & \multirow[t]{3}{*}{$0.820(0.723-0.902)$} & 3.6 & 76.5 & 82.1 & 0.59 & 4.3 & 0.3 \\
\hline & & 4.2 & 71.4 & 85.7 & 0.57 & 5.0 & 0.3 \\
\hline & & 3.9 & 73.9 & 82.1 & 0.56 & 4.1 & 0.3 \\
\hline \multirow[t]{3}{*}{ Medial parapatellar } & \multirow[t]{3}{*}{$0.776(0.677-0.864)$} & 3 & 66.4 & 85.7 & 0.52 & 4.6 & 0.3 \\
\hline & & 2.7 & 69.7 & 82.1 & 0.52 & 3.9 & 0.4 \\
\hline & & 3.3 & 62.2 & 85.7 & 0.48 & 4.3 & 0.4 \\
\hline \multirow[t]{3}{*}{ Lateral parapatellar } & \multirow[t]{3}{*}{$0.789(0.692-0.877)$} & 2.7 & 77.3 & 71.4 & 0.49 & 2.7 & 0.4 \\
\hline & & 3.3 & 68.9 & 78.6 & 0.48 & 3.2 & 0.4 \\
\hline & & 3 & 74.8 & 71.4 & 0.46 & 2.6 & 0.4 \\
\hline \multicolumn{8}{|l|}{$30^{\circ}$} \\
\hline Suprapatellar & $0.849(0.770-0.917)$ & 6 & 66.4 & 92.9 & 0.59 & 9.4 & 0.4 \\
\hline & & 3.6 & 86.6 & 71.4 & 0.58 & 3.0 & 0.2 \\
\hline & & 4.8 & 79.0 & 78.6 & 0.58 & 3.7 & 0.3 \\
\hline Medial parapatellar & $0.791(0.697-0.870)$ & 3.3 & 63.0 & 89.3 & 0.52 & 5.9 & 0.4 \\
\hline & & 3.9 & 58.8 & 92.9 & 0.52 & 8.3 & 0.4 \\
\hline & & 3.6 & 61.3 & 89.3 & 0.51 & 5.7 & 0.4 \\
\hline Lateral parapatellar & $0.774(0.678-0.857)$ & 4.2 & 59.7 & 85.7 & 0.45 & 4.2 & 0.5 \\
\hline & & 3.3 & 71.4 & 71.4 & 0.43 & 2.5 & 0.4 \\
\hline & & 3 & 74.8 & 67.9 & 0.43 & 2.3 & 0.4 \\
\hline $45^{\circ}$ & & & & & & & \\
\hline Suprapatellar & $0.840(0.759-0.909)$ & 3.6 & 84.9 & 75.0 & 0.60 & 3.4 & 0.2 \\
\hline & & 3.9 & 81.5 & 75.0 & 0.57 & 3.3 & 0.2 \\
\hline & & 5.1 & 68.9 & 85.7 & 0.55 & 4.8 & 0.4 \\
\hline Medial parapatellar & $0.707(0.611-0.797)$ & 3.3 & 52.1 & 85.7 & 0.38 & 3.6 & 0.6 \\
\hline & & 2.7 & 61.3 & 75.0 & 0.36 & 2.5 & 0.5 \\
\hline & & 3 & 58.8 & 75.0 & 0.34 & 2.4 & 0.5 \\
\hline Lateral parapatellar & $0.766(0.664-0.855)$ & 3.6 & 63.0 & 78.6 & 0.42 & 2.9 & 0.5 \\
\hline & & 3.3 & 75.6 & 85.7 & 0.61 & 5.3 & 0.3 \\
\hline & & 3.6 & 71.4 & 89.3 & 0.61 & 6.7 & 0.3 \\
\hline $60^{\circ}$ & & & & & & & \\
\hline Suprapatellar & $0.843(0.767-0.909)$ & 3.3 & 75.6 & 85.7 & 0.61 & 5.3 & 0.3 \\
\hline & & 3.6 & 71.4 & 89.3 & 0.61 & 6.7 & 0.3 \\
\hline & & 3.9 & 66.4 & 92.9 & 0.59 & 9.4 & 0.4 \\
\hline Medial parapatellar & $0.710(0.619-0.794)$ & 2.4 & 54.6 & 85.7 & 0.40 & 3.8 & 0.5 \\
\hline & & 2.1 & 56.3 & 82.1 & 0.38 & 3.1 & 0.5 \\
\hline & & 1.8 & 58.0 & 78.6 & 0.37 & 2.7 & 0.5 \\
\hline Lateral parapatellar & $0.740(0.650-0.822)$ & 3.9 & 48.7 & 96.4 & 0.45 & 13.5 & 0.5 \\
\hline & & 2.7 & 63.0 & 78.6 & 0.42 & 2.9 & 0.5 \\
\hline & & 4.5 & 43.7 & 96.4 & 0.40 & 12.1 & 0.6 \\
\hline $90^{\circ}$ & & & & & & & \\
\hline Suprapatellar & $0.829(0.752-0.895)$ & 2.1 & 72.3 & 89.3 & 0.62 & 6.8 & 0.3 \\
\hline & & 2.4 & 67.2 & 92.9 & 0.60 & 9.5 & 0.4 \\
\hline & & 2.7 & 65.5 & 92.9 & 0.58 & 9.2 & 0.4 \\
\hline Medial parapatellar & $0.728(0.641-0.808)$ & 0.9 & 58.0 & 85.7 & 0.44 & 4.1 & 0.5 \\
\hline & & 1.2 & 55.5 & 85.7 & 0.41 & 3.9 & 0.5 \\
\hline & & 0.6 & 58.8 & 82.1 & 0.41 & 3.3 & 0.5 \\
\hline Lateral parapatellar & $0.785(0.701-0.861)$ & 2.1 & 64.7 & 85.7 & 0.50 & 4.5 & 0.4 \\
\hline & & 1.8 & 67.2 & 82.1 & 0.49 & 3.8 & 0.4 \\
\hline & & 2.4 & 57.1 & 89.3 & 0.46 & 5.3 & 0.5 \\
\hline
\end{tabular}

* AUC-ROC $=$ area under the receiver operator characteristic curve; $95 \%$ CI $=95 \%$ confidence interval; LR $+=$ positive likelihood ratio; LR $-=$ negative likelihood ratio. 

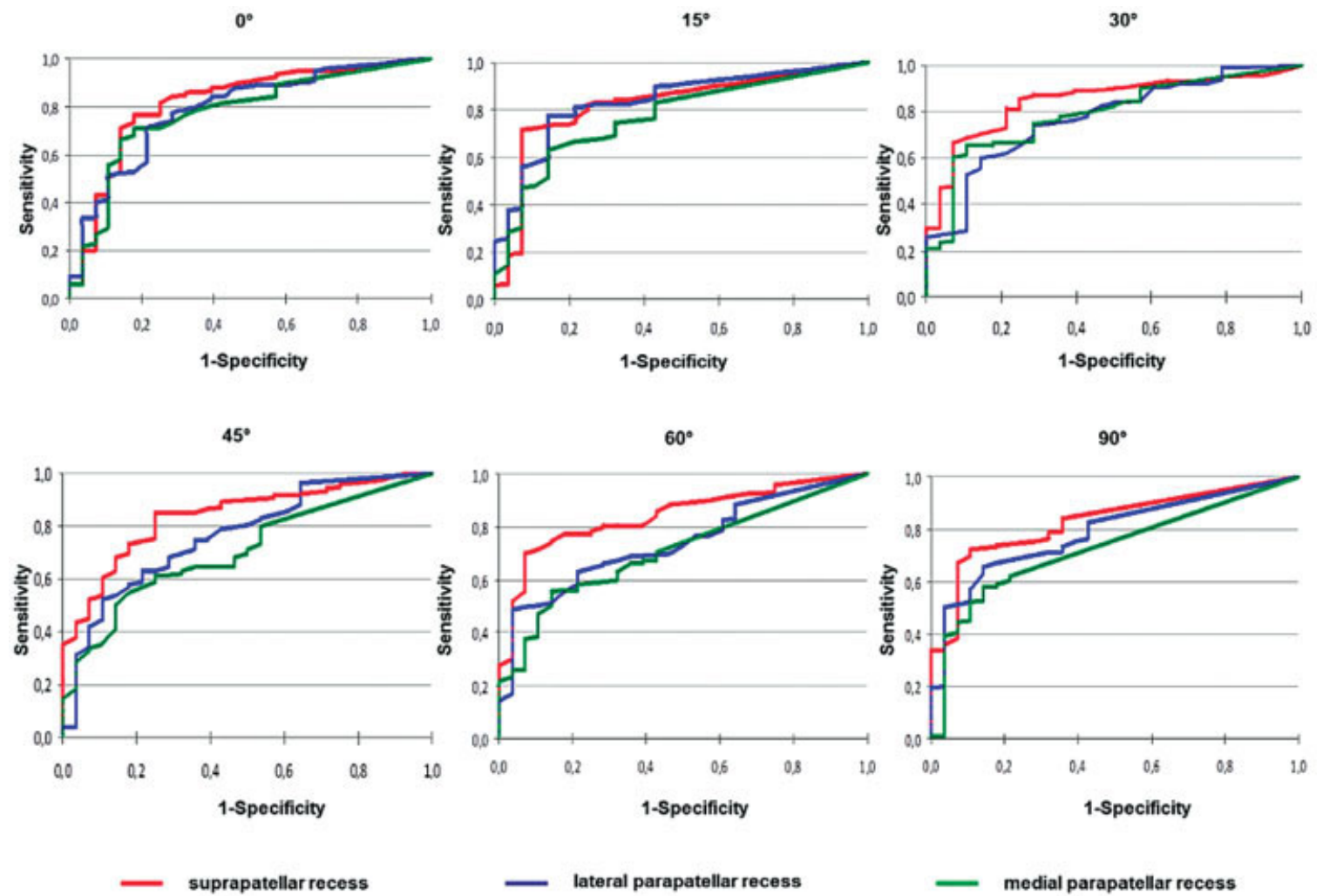

Figure 3. Receiver operating curves for synovial fluid measurement of the suprapatellar, medial parapatellar, and lateral parapatellar recesses of the knee at varying degrees of flexion of the knee joint, corresponding to the 18 logistic models in Table 1.

$10^{-16}$ ), and considering the combination of knee and transducer position $\left(P \leq 10^{-4}\right)$. Supplementary analysis using Tukey's test revealed that, in particular, the most significant difference between measures was observed comparing $30^{\circ}-0^{\circ}$, followed by $45^{\circ}-0^{\circ}$ and $15^{\circ}-0^{\circ}$ (no statistical difference between these 2 positions), and by $60^{\circ}-0^{\circ}$ and $90^{\circ}-0^{\circ}$ (no statistical difference between these 2 positions) independently of the recess examined. Considering the variability due to the recess position, the difference between measures was significantly higher in the suprapatellar recess as compared to the lateral parapatellar and medial parapatellar recesses $\left(P \leq 10^{-8}\right)$, independently of the degree of knee flexion. Tukey's test showed that when using $0^{\circ}$ as reference, the magnitude of change of sagittal diameter in the 3 examined recesses was significantly higher at $30^{\circ}$ and $45^{\circ}$ of flexion in each recess (Figure 2).

ROC analysis confirmed these results and revealed that the combination of suprapatellar scan at the $30^{\circ}$ position was the best knee and transducer position for detecting knee effusion, characterized by the largest AUC and therefore the best discriminative power (Table 1 and Figure 3). Logistic regression models were performed to establish the relationship between the presence of effusion depending on each synovial fluid measure. Cutoff points were determined to predict effusion by the Youden index maximum for each model. The best cutoff value favoring this recess and at this degree of flexion when favoring high sensitivity was $3.6 \mathrm{~mm}$ (corresponding to a sensitivity of $86.6 \%$ and a specificity of $71.4 \%$ ) and $6 \mathrm{~mm}$ when favoring high specificity (corresponding to a sensitivity of $66.4 \%$ and a specificity of $92.9 \%$ ) (Table 1). As shown by the AUC confidence intervals and bootstrap results, there was no statistical difference between the AUC for the suprapatellar recess at $30^{\circ}$ of flexion and that of the lateral parapatellar recess at $0^{\circ}$ of flexion. No differences were observed among the different combinations of angles for determining the final ultrasound diagnosis of knee abnormality.

\section{DISCUSSION}

Various approaches and methods with regard to both the clinical and ultrasound examination of the knee are used in daily practice to better detect synovial effusion. The beneficial effect of voluntary quadriceps contraction on the sensitivity of fluid detection in the suprapatellar recess has been demonstrated (7). However, the quadriceps muscle is known to be inhibited by joint effusion that is influenced both by the volume of the effusion and the position of the joint (14). This study was performed in order to investigate which scanning position provides the best information about synovial fluid in the knee using ultrasound. By evaluating the sagittal diameter of synovial fluid in the 3 major recesses of the knee, we were able to show that it increased in all the recesses with the knee in the flexed position as compared to the neutral, extended po- 
sition. The fact that fluid diameter decreased at higher degrees of flexion might be due to changes in intraarticular pressure, which were shown to be highest at full flexion or extension of the knee joint $(14,15)$. Several earlier studies have assessed cutoff in asymptomatic joints of a large number of healthy subjects $(16,17)$. In these studies, the cutoff values for normal synovial fluid in the suprapatellar recess were between $2.2-4.9 \mathrm{~mm}$; however, the studies differed in the mode of examination (the latter study measured synovial fluid in the extended position during quadriceps contraction). The obtained cutoff values in our study were found to be generally similar to those demonstrated in these earlier studies and was found to be between 3.6-6.0 $\mathrm{mm}$ in the combination yielding the highest AUC (suprapatellar recess, $30^{\circ}$ of flexion of the knee), depending on whether sensitivity or specificity was favored (Table 1). The lateral recess at $0^{\circ}$ of flexion of the knee also performed quite similarly. However, the sonographers observed less variation in fluid diameter during various degrees of flexion when scanning the lateral recess, as compared to the suprapatellar recess. This was likely due to the larger surface of the latter as opposed to the former, which led to larger variation in sagittal diameter. We therefore propose starting the examination of the knee in the extended position; if fluid above the cutoff is detected in the lateral parapatellar recess, the examiner need not proceed further with the examination. However, if this is not the case, we would advise that an examination of the suprapatellar recess at $30^{\circ}$ of flexion of the knee be performed as this position permits the best detection of even small amounts of fluid.

Every knee and scan position has distinct cutoff values to detect abnormal effusion and to aid in globally evaluating effusion and synovial proliferation. A further study examining the posterior recess and potential Baker's cyst may allow us to further elaborate the changes in effusion quantity. The fact that no difference could be demonstrated between degrees of flexion for predicting final abnormality of the examined knee can be explained by the setup of the study, which featured the sonographers judging final abnormality after completing the ultrasound evaluation. Scanning the 3 recesses of the knee in both the flexed and extended position will allow the sonographer to gain a better understanding of the distribution of effusion within the knee joint. Our results suggest that the measurement of synovial fluid in the knee in the suprapatellar scan with the knee flexed to $30^{\circ}$ allows the most optimal detection of even minimal amounts of abnormal joint effusion in agreement with recent findings obtained in salineinjected cadavers (18). Performing a dynamic ultrasound examination may also influence the physician to change the anatomic diagnosis of involved structures in certain musculoskeletal conditions and, consequently, the planning of joint injections and that of overall treatment $(19,20)$. Such modifications were associated with a trend towards improved short-term symptomatic treatment effect by rheumatologists (20). This can be easily performed with the use of a standard goniometer and does not significantly lengthen the ultrasound examination. Full evalua- tion of the knee, including the examination of the 3 recesses in the extended position and during the dynamic examination of the knee (knee flexed from $0-90^{\circ}$ ) provides the most information regarding the presence and distribution of synovial effusion to the sonographer.

\section{AUTHOR CONTRIBUTIONS}

All authors were involved in drafting the article or revising it critically for important intellectual content, and all authors approved the final version to be submitted for publication. Dr. Mandl had full access to all of the data in the study and takes responsibility for the integrity of the data and the accuracy of the data analysis.

Study conception and design. Mandl, Brossard, Backhaus, Bruyn, Chary-Valckenaere, Iagnocco, Filippucci, Jousse-Joulin, Möller, Naredo, Wakefield, D'Agostino, Balint.

Acquisition of data. Mandl, Backhaus, Bruyn, Chary-Valckenaere, Iagnocco, Freeston, Gandjbakhch, Jousse-Joulin, Möller, Naredo, Schmidt, Szkudlarek, Terslev, Wakefield, Zayat, D’Agostino, Balint.

Analysis and interpretation of data. Mandl, Brossard, Aegerter, Chary-Valckenaere, Iagnocco, Jousse-Joulin, Möller, Naredo, Wakefield, D’Agostino, Balint.

\section{REFERENCES}

1. Kane D, Balint PV, Sturrock RD. Ultrasonography is superior to clinical examination in the detection and localization of knee joint effusion in rheumatoid arthritis. J Rheumatol 2003; 30:966-71.

2. Fam AG, Wilson SR, Holmberg S. Ultrasound evaluation of popliteal cysts on osteoarthritis of the knee. J Rheumatol 1982;9:428-34.

3. Hauzeur JP, Mathy L, De Maertelaer V. Comparison between clinical evaluation and ultrasonography in detecting hydrarthrosis of the knee. J Rheumatol 1999;26:2681-3.

4. Cellerini M, Salti S, Trapani S, D’Elia G, Falcini F, Villari N. Correlation between clinical and ultrasound assessment of the knee in children with mono-articular or pauci-articular juvenile rheumatoid arthritis. Pediatr Radiol 1999;29:117-23.

5. Riente L, Delle Sedie A, Filippucci E, Scire CA, Iagnocco A, Gutierrez M, et al. Ultrasound imaging for the rheumatologist. XXVII. Sonographic assessment of the knee in patients with rheumatoid arthritis. Clin Exp Rheumatol 2010;28:300-3.

6. Backhaus M, Burmester GR, Gerber T, Grassi W, Machold KP, Swen WA, et al, for the Working Group for Musculoskeletal Ultrasound in the EULAR Standing Committee on International Clinical Studies including Therapeutic Trials. Guidelines for musculoskeletal ultrasound in rheumatology. Ann Rheum Dis 2001;60:641-9.

7. Ike RW, Somers EC, Arnold EL, Arnold WJ. Ultrasound of the knee during voluntary quadriceps contraction: a technique for detecting otherwise occult effusions. Arthritis Care Res (Hoboken) 2010;62:725-9.

8. Fenn S, Datir A, Saifuddin A. Synovial recesses of the knee: MR imaging review of anatomical and pathological features. Skeletal Radiol 2009;38:317-28.

9. Rhodes LA, Grainger AJ, Keenan AM, Thomas C, Emery P, Conaghan PG. The validation of simple scoring methods for evaluating compartment-specific synovitis detected by MRI in knee osteoarthritis. Rheumatology (Oxford) 2005;44:1569-73.

10. Rhodes LA, Keenan AM, Grainger AJ, Emery P, McGonagle D, Conaghan PG. The relationship between limited MRI section analyses and volumetric assessment of synovitis in knee osteoarthritis. Clin Radiol 2005;60:1295-9.

11. Boldt JG, Munzinger UK, Zanetti M, Hodler J. Arthrofibrosis associated with total knee arthroplasty: gray-scale and power Doppler sonographic findings. Am J Roentgenol 2004;182: 337-40.

12. Rubaltelli L, Fiocco U, Cozzi L, Baldovin M, Rigon C, Bortoletto P, et al. Prospective sonographic and arthroscopic eval- 
uation of proliferative knee joint synovitis. J Ultrasound Med 1994;13:855-62.

13. Wakefield RJ, Balint PV, Szkudlarek M, Filippucci E, Backhaus M, D'Agostino MA, et al. OMERACT 7 Special Interest Group. Musculoskeletal ultrasound including definitions for ultrasonographic pathology. J Rheumatol 2005;32:2485-7.

14. Jensen K, Graf BK. The effects of knee effusion on quadriceps strength and knee intraarticular pressure. Arthroscopy 1993; 9:52-6.

15. Alexander C, Caughey D, Withy S, van Puymbroeck E, Munoz D. Relation between flexion angle and intraarticular pressure during active and passive movement of the normal knee. J Rheumatol 1996;23:889-95.

16. Van Holsbeeck MV, Introcaso JH, editors. Musculoskeletal ultrasound. Vol. 1. St. Louis: Mosby; 2001. p. 315-9.
17. Schmidt WA, Schmidt H, Schicke B, Gromnica-Ihle E. Standard reference values for musculoskeletal ultrasonography. Ann Rheum Dis 2004;63:988-94.

18. Hong BY, Lim SH, Cho YR, Kim HW, Ko YJ, Han SH, et al. Detection of knee effusion by ultrasonography. Am J Phys Med Rehabil 2010;89:715-21.

19. D’Agostino MA, Ayral X, Baron G, Ravaud P, Breban M, Dougados M. Impact of ultrasound imaging on local corticosteroid injections of symptomatic ankle, hind-, and mid-foot in chronic inflammatory diseases. Arthritis Rheum 2005;53: 284-92.

20. Karim Z, Wakefield RJ, Conaghan PG, Lawson CA, Goh E, Quinn MA, et al. The impact of ultrasonography on diagnosis and management of patients with musculoskeletal conditions. Arthritis Rheum 2001;44:2932-3. 